\title{
El sistema constitucional de Roma, capital deltalia
}

\section{Pietro Barrera*}

Roma es, junto con Atenas, la más antigua y noble de todas las capitales europeas, pero en realidad se trata de una capital muy joven si se consideran su rol como símbolo de la unidad política de la nación y su función actual como sede de los pode res públicos. Tan sólo han pasado 132 años desde que Roma se integró en el Reino de Italia y ha visto el fin del poder temporal del Papa. La relación entre Roma e Italia ha sido tormentosa, contradictoria y marcada por unas enormes expectativas simbólicas y el recelo. Camilo Cavour, el primer ministro piamontés y constructor de la unificación nacional, se dirigía de la siguiente forma al Parlamento en Turín, mientras abogaba por Roma como potencial capital de Italia. «Ahora, señores, Roma reúne todas las circunstancias históricas, intelectuales y morales que han de determinar las condiciones de una gran nación. Roma es la única ciudad de I talia que no ha tenido un pasado municipal exclusivo; toda la historia de Roma, desde los Césares hasta el día de hoy, ha sido la historia de una ciudad cuya importancia se extiende mucho más allá de su territorio; de una ciudad, por lo tanto, destinada a ser la capital de una gran nación». A pesar de esto, después de re conquistada «Su capital» en 1870, Italia se vio obligada a darle un status jurídico, organizativo y funcional similar a las capitales de otras grandes naciones europeas.

El Reino de Italia, en sus primeras décadas de existencia, sólo llevó a cabo medidas para modernizar las infraestructuras de la ciudad y permitir su propio desarrollo: la «fiebre de la vivienda» que invadió Roma después de 1880, con un vínculo sin precedentes entre los nuevos potentados financieros y las viejas rentas de la tierra, dejaron en poco tiempo un desolador escenario de estragos medioambientales, sociales y económicos. La mayor preocupación de estas nuevas clases dirigentes parecía ser «mantener a Roma en el más absoluto orden y máxima tranquilidad, especialmente cuando el Parlamento se hallaba en sesión» (éstas eran las palabras de un M inistro, el señor Lanza, en 1873).

Fue el fascismo, en cambio, el que planteó el problema del sistema jurídico de la capital de una manera fuertemente simbólica. En absoluta sintonía con un modelo de Estado autoritario y centralizado, el Régimen decidió transformar Roma en un $\mathrm{G}$ overnatorate y confió su mandato a un «gobernador» nombrado por el Gobierno. Es probable que la fuerte experiencia del $\mathrm{G} 0$ vernatorate - donde la «Retórica Romana» apoyó la violenta supresión de toda autonomía local - pueda explicar las dificultades encontradas, en las primeras décadas posteriores a la Constitución de 1948, para admitir para Roma, capital de la nueva República, un status diferenciado y especial, aunque completamente democrático.

0 tra fuente de indecisión, derivada de la peculiar condición de «ciudad eterna», como sede del Supremo Pontífice, se añadió a todo esto. El Tratado de Letrán de 1929 acabó con el «pleito romano» (la disputa entre el Reino de Italia y el Santo Padre), comprometiéndose el Estado Italiano a reconocer la «naturaleza sagrada» de Roma y a prohibir todo aquello que contrastara con dicha natural eza dentro de la ciudad. La extraordinaria peculiaridad de la ciudad - siendo la capital de un moderno Estado de mocrático, el centro de gravedad de una I glesia universal, un centro cultural único en el mundo (y, durante cincuenta años, sede de al gunas de las más importantes agencias de las $\mathrm{N}$ aciones Unidas) - terminó por obstaculizar su desarrollo, su modernización institucional y la completa racionalización de su forma de gobierno.

La Constitución Republicana, aprobada el 1 de enero de 1948, no hace referencia al guna a la capital, pero jafirma reconocer el valor fundamental del Tratado de Letrán! N 0 hay sorpresas 
en las décadas siguientes, ninguno o pocos esfuerzos se realizaron para proveer a la capital de la República de un sistema jurídico y financiero apropiado a sus nuevas necesidades. D e hecho, una vez que se restablecieron las reglas de la democracia local, a Roma se le reconocieron las mismas funciones, organización y autonomía que a cualquier otra ciudad del país, basándose en el principio de «igualdad de trato» entre autoridades locales, que se remontaba a la legislación italiana de 1859, la Ley Ratazzi del Reino de C erdeña, y que tenía su fundamento en el artículo 128 de la Constitución. En la práctica se estableció una costumbre distorsionada y distorsionante, y sus consecuencias todavía son hoy evidentes, por la cual se necesitaron leyes especiales para grandes eventos (los Juegos O límpicos de 1960, el M undial de Fútbol de 1990 y el Año Jubileo del 2000) para conseguir los re cursos necesarios para la modernización de la capital, que estaba incluso en desventaja con otras grandes ciudades en las transfe rencias de ingresos per cápita.

A decir verdad, en la primera legislatura del Parlamento Re publicano (1948-1953) se presentaron «proyectos de ley para la capital» por partidos políticos opuestos. El Partido D emócrata Cristiano puso el acento en encargar al M inistro del Interior la función, que por lo demás se atribuía a los Prefectos, de supervisar sustancialmente las instituciones locales; la propuesta de la izquierda se movía en la dirección contraria y pretendía ofrecer mayores oportunidades de autogobierno, con la previsión de consejos de distrito en distintas partes de la ciudad. $N$ ada de esto se hizo (la descentralización inframunicipal comenzó experimentalmente en Roma en 1966 y sólo se reguló por la Ley de Grandes Ciudades en 1976). El único destello de conciencia apareció en la legislación de 1953, que reconocía una modesta contribución financiera anual para la ciudad de Roma «por las molestias que la ciudad soporta por ser la capital de la República». Las sumas de dinero eran realmente exiguas, pero, a pesar de esto, subrayaban un punto de gran importancia: las funciones propias de una capital conllevan un coste, cargan la vida diaria de la ciudad con molestias y no es posible ni justo que la comunidad local tenga que cargar con todo por sí misma.

La innovación más importante tuvo lugar en 1990, cuando el Parlamento aprobó la Ley 396, para la implementación de un «Programa para Roma, Capital de Italia», sin precedentes. La Ley pretendía financiar un plan plurianual de obras públicas; sin embargo, su lógica intrínseca era profundamente diferente de las leyes de finales del siglo xıx. En el centro del sistema se situaba un nuevo órgano, la «Comisión de Roma, capital de Italia». Estaba presidida por el Jefe del Gobierno y compuesta por cuatro importantes ministerios, el Alcalde de Roma, el Presidente de la Provincia y el Presidente de la Región, y estaba encargada de la armonización de las necesidades y los puntos de vista, y de encontrar la necesaria convergencia entre los diferentes niveles de gobierno. El programa de trabajo se desarrollaba con la coopera- ción de todo el Estado y las Administraciones Locales implicadas, pero era en todo caso el Ayuntamiento el que tenía la última palabra, y para las leyes de ese tiempo los procedimientos administrativos resultantes se modernizaron y se racionalizaron.

El modelo resultante se vio frustrado a causa de los insuficientes recursos disponibles, por la fragmentación de los trabajos sin prioridades claras y por el rápido «envejecimiento» de los procedimientos, que llegaron a ser en poco tiempo intolerablemente voluminosos. El modelo había permitido fijar, de todos modos, tres puntos importantes para el futuro desarrollo del sistema. Ante todo, el firme reconocimiento de la centralidad de la autonomía local: nadie puede pensar en una vuelta atrás a los modelos autoritarios de governatorate, ni a las difíciles experiencias históricas de ciudades como París o Washington, que han esperado durante décadas para ver reconocido su derecho de autogobierno. En segundo lugar, el principio de iniciativa institucional concertada: si toda capital es por definición una ciudad «normal», donde viven ciudadanos con expectativas y derechos normales, y una ciudad «especial», donde se desempeñan funciones de interés nacional (y, en Roma, incluso de interés supranacional), es necesario encontrar para todos los niveles de gobierno - el Estado, la ciudad y los niveles intermedios- lugares y herramientas con las que puedan confrontar, colaborar y armonizar sus necesidades respectivas. $Y$, eventualmente, la planificación: Roma no puede «regirse al día», es con mucho una ciudad compleja y difícil, necesita programas a largo plazo y recursos financieros estables distribuidos a lo largo del tiempo.

Claramente, ese no fue el caso cuando se tomaron prestados los instrumentos organizativos y jurídicos de la «Ley para Roma, Capital de Italia» de 1990, para la preparación del Gran Jubileo del año 2000, y se añadió un nuevo elemento de gran importancia práctica: por vez primera se introdujo un órgano mixto - República Italiana/Ciudad del Vaticano- en 1999 para tratar con los problemas organizativos y logísticos de la ciudad. Y, por primera vez, el Alcalde de Roma entraba legalmente en un foro bilateral (de D erecho internacional).

Brevemente, éste es el cuadro histórico y jurídico que contempla la emergencia de una nueva regulación constitucional, con la Ley Constitucional 3/2001. Dicha regulación establece que «Roma es la capital de la República. La Ley del Estado regula su sistema». La reforma fue objeto de un referéndum el 7 de octubre de 2001 y obtuvo la aprobación explícita de 500.000 romanos (aquel día era peculiar: el comienzo de los bombardeos sobre Afganistán, jcuando las mentes y las emociones se hallaban concentradas en otros problemas!). La somera regulación constitucional contiene dos declaraciones diferentes e igual mente importantes. La primera - Roma es la capital de la República- podría parecer superficial e incluso superflua si no fuese por el hecho de que el Parlamento la aprobó durante un conflicto político altamente simbólico, que fue estimulado por la denominada Lega 
N ord (Liga N orte). Explícitos pulsos separatistas parecían desafiar la unidad nacional, en el nombre de una supuesta «identidad del Po», y Roma se encontró a sí misma, de modo indeseado, haciendo el papel de «viejo poder». La campaña alcanzó puntos severos («Roma ladrona, la Lega ti bastona») y, en el Parlamento esta formación política presentó decenas de enmiendas intentando identificar otras tantas «capitales» en pequeños centros del Norte de Italia. La solemne proclamación de la función nacional de Roma ha sido una «rotunda» y rebatida elección, que estaba conectada explícitamente con el proceso de transformación federal que el país estaba a punto de acometer. Además, no es extraño que, entre las Constituciones contemporáneas, las de los países con un sistema federal necesiten definir la identidad y el rol de sus capitales: la capital adopta el papel simbólico del pacto de unidad entre los distintos componentes de la federación.

La segunda declaración constitucional - la Ley del Estado regula el sistema de la capital- posee un valor práctico e inmediato. D e hecho, por una parte, ayudó a superar las trabas opre sivas e irracionales del principio de «igualdad de trato», que dejó a Roma en el mismo nivel que el municipio más pequeño deltalia durante 130 años y, por otra parte, permitió confiar al Estado, y no a la Región del Lazio, el control del sistema de la capital (organización y funciones). La regulación constitucional expre sada de forma concisa permite sentar los puntos esenciales del «status de capital»:

a) La ubicación dentro del artículo 114, después del primer ( «a República se compone de M unicipios, Provincias, ciudades metropolitanas, Regiones y el Estado») y segundo apartados, deja claro que Roma no puede lisa y llanamente ser identificada con esas «formas» de comunidades territoriales; la regulación constitucional no atribuye el título de capital de la República ni al «M unicipio de Roma» ni al Área M etropolitana de Roma, sino simple mente a Roma, a la ciudad de Roma.

b) La regulación abre el Capítulo $V$ de la Constitución y deja claro que la ley del sistema de la capitalidad puede derogar todas las regulaciones del sistema de autonomías territoriales: por tanto, la Constitución ha confiado a la ley del Estado los asuntos que son gestionados por un amplio sistema de fuentes en otras comunidades territoriales (la misma Constitución, las leyes del Estado, los Estatutos y leyes regionales, los estatutos y normas para la autonomía).

c) Más aún: el núcleo del tercer apartado del artículo 114 es una norma de demarcación, que tiene el mismo valor que las normas constitucionales sucesivas que dividen las responsabilidades legislativas en tres subgrupos de la autoridad exclusiva del Estado, compartidas y de la autoridad exclusiva de las Regiones. Si el «sistema de la capital» no es sólo una «competencia», sino más bien un sistema de subcompetencias relacionadas con aspectos organizativos y funcionales de la capital, su control, por norma constitucional, estará reservado tan sólo a la ley estatal.

d) Además, si esto no fuese así, la «Ley de la Capital» sería sustancialmente inútil, desde el momento en que, de cualquier modo, depende de la ley del Estado el control de la forma de gobierno («ley electoral y órganos de gobierno») y las «funciones fundamentales» de todas las autoridades locales (artículo 117.2 letra p). Además de esto, una vez que se ha superado el principio de homogeneidad y se ha constitucionalizado el principio de diferenciación, sería posible que una ley del Estado estableciese un control diferenciado para la ciudad de Roma, posiblemente a causa de su dimensión demográfica, que es totalmente anómala en el ámbito de las ciudades italianas.

e) La cláusula de reserva en favor del Estado del artículo 114, apartado tercero, es perfectamente coherente e incluso esencial en esta cuestión del «sistema de la capital», que demanda una competencia especial del Estado (podríamos llamarla una interferencia actual) en la organización y el control de las funciones de su territorio, como «C apital de la República», que se encuentra situada al servicio de una compleja situación institucional constituida por el Estado, todas las Regiones, las Provincias, las ciudades M etropolitanas y los M unicipios. El control de la capital se confía a una ley estatal, puesto que es la fuente subconstitucional de interés general que afecta a todosy surge de la entera «comunidad» de la República.

f) Muchos ejemplos de comparación entre los sistemas de otros países democráticos occidentales admiten la ratio de esta opción, que aparentemente no respeta la autonomía local tal como es entendida; pero esos mismos ejemplos muestran cómo el orden jurídico de la capital ha respetado el derecho de la comunidad local al autogobierno como esencial para la gestión de sus «intereses normales», después de un desarrollo institucional que ha llevado más de un siglo en algunos casos. Por tanto, la capital como tal es objeto de un control legislativo especial por parte del Estado, y como «ciudad normal» se halla investida de las competencias y prerrogativas típicas de otras comunidades locales.

g) Además, con el fin de obtener funciones altamente efectivas propias de una capital, sería útil y necesario atribuir a las instituciones locales potestades autónomas particularmente notables. La entidad local no tiene, por lo demás, un derecho incomprensible a una mayor autonomía local en comparación con otras entidades, sino que las instituciones locales pueden poseer mayores potestades 
autónomas, siempre y cuando se encaminen al cumplimiento efectivo de los propósitos propios de la capital de la República.

h) El objeto de la cláusula de reserva introduce un límite: un principio general de racionalidad nos conduce a creer que el sistema de Roma derogará la regulación de las otras entidades territoriales, en aquello en que las derogaciones estén conectadas y encaminadas hacia las funciones propias o típicas de la capital.

i) Para los asuntos que no tienen una relación teleológica ni funcional con la «capitalidad» de Roma, el sistema hará alusión per relationem al control constitucional y legislativo de otras entidades territoriales (evidentemente tanto de los municipios como de las ciudades metropolitanas, las dos «formas» en que la Constitución organiza los centros urbanos).

j) El hecho de que Roma está dentro de la Región del Lazio implica evidentemente que se aplican en su territorio las previsiones del Estatuto de la Región, las leyes y reglamentos, porque no han sido derogados por el peculiar sistema de capitalidad.

Ahora, es el turno del Parlamento. El 28 de febrero del 2002, el Alcalde de Roma, Walter Veltroni, presentó una hipótesis de trabajo al Presidente del Consejo de M inistros, a los Presidentes de las Cámaras y a todos los diputados elegidos en Roma. No es realmente una proposición de ley, puesto que la administración de la ciudad no posee capacidad de iniciativa legislativa, sino una serie de propuestas y objetivos, que el Alcalde presentó al Gobierno y al Parlamento, para que éstos la transformen en una ley.

De acuerdo con las condiciones constitucionales que hemos indicado, la «peculiaridad» del sistema de capitalidad responderá a un criterio general de racionalidad y razonabilidad, para dife renciarse de los sistemas de otras grandes ciudades (ciudades metropolitanas, en el nuevo contexto constitucional) por las manifiestas peculiaridades de la ciudad de Roma que se derivan de:

a) La presencia de los órganos constitucionales del Estado y las funciones típicas conectadas a cualquier capital moderna, comenzando por el papel que una capital está llamada a jugar como símbolo de la unidad nacional.

b) La presencia del Santo Padre y de las más importantes instituciones internacionales comprometidas en la lucha contra el hambre y la pobreza (FAO, IFAD, WFD PAM ), y el correspondiente compromiso con el Derecho internacional asumido por Italia.

a) El patrimonio histórico y artístico, que es único por su valor, complejidad y extensión territorial.
El sistema de la capital se orientará, entonces, a la armonización de los intereses de las comunidades local y nacional y, además, a desarrollar la colaboración entre las instituciones locales, las instituciones estatales y las de la Región del Lazio, con el fin de garantizar:

a) El desarrollo económico y social de la capital de la República; la calidad del desarrollo urbano; la protección y la promoción de su patrimonio histórico, artístico y medioambiental, y el fortalecimiento de su prestigio y su identidad internacional.

b) Los servicios urbanos necesarios para el funcionamiento de los órganos constitucionales del Estado y los órganos y dependencias públicas nacionales, así como con vistas a favorecer la participación de los ciudadanos y las organizaciones sociales en la vida institucional, política, económica y social del país, así como los servicios urbanos nece sarios para la funcionalidad de las delegaciones extranjeras e instituciones internacionales con sede en Roma, así como respecto al Estado de la Ciudad del Vaticano.

c) El acceso a los niveles esenciales de servicios sociales para los residentes así como para todos los beneficiarios de dichos servicios en la ciudad de Roma (turistas, peregrinos de San Pedro, ciudadanos que acceden a las oficinas y órganos presentes en la capital).

d) La identificación de los recursos financieros adicionales necesarios para cubrir necesidades diferentes de las que tiene normalmente cualquier comunidad local (en perfecta sintonía con las previsiones del artículo 119 de la Constitución).

Con el fin de alcanzar estos objetivos y cubrir las necesidades de la capital (o mejor dicho, jde la República para su capital!), las instituciones de la ciudad de Roma han de desempeñar funciones administrativas para cuestiones «estratégicas» con potestades autónomas irrelevantes. El punto esencial de estas «potestades autónomas» podría ser un poder especial de regulación que permitiese a la capital adoptar decisiones en al gunas cuestiones cruciales para su desarrollo y buen funcionamiento, derogando al gunas regulaciones fijadas por las leyes estatales o regionales. La propuesta presentada por el Alcalde recuerda la última «norma de desregulación», mencionada en el artículo 17, párrafo segundo, de la Ley 400/1988. La hipótesis propuesta es que tales normas son posibles, evidentemente sólo en relación con los asuntos que no están cubiertos por la cláusula de reserva absoluta, previa la determinación, por la ley, de los principios fundamentales (i.e. en armonía con los principios fundamentales establecidos por otras leyes vigentes), y a través de un proceso de agregación que implica al Comité para Roma, Capital de Italia y, además, al Presidente del Consejo de M inistros y al Presidente de la Región. Como resultado, los asuntos para los cuales la au- 
tonomía reguladora podría, o más bien debería, ser implementada son los siguientes:

a) La administración del territorio y el control de la planificación urbana.

b) La protección del medio ambiente, incluyendo procedimientos para la valoración del impacto ambiental y la salvaguarda del patrimonio histórico y cultural.

c) Planificación y puesta en marcha de infraestructuras públicas de interés nacional.

d) El funcionamiento de redes e infraestructuras de transporte público.

e) El control del tráfico rodado.

f) La promoción del desarrollo económico.

g) La organización de los servicios sociales y los servicios de protección de la salud.

h) Las normas de la policía local.

Por otra parte, en relación con su comunidad local, las instituciones de la ciudad de Roma habrán de desempeñar de forma clara todas las funciones propias de los municipios y de las ciudades metropolitanas, siempre y cuando tenga lugar el proceso de agregación entre el municipio de Roma y otros municipios, tal como prescribela ley.

El «estatuto institucional» de la capital puede implicar regulaciones financieras, tales como la transferencia a la capital de los recursos adecuados para desempeñar las funciones que no estén relacionadas con las necesidades normales de la entidad local. En este sentido, la ley se hallaría en perfecta sintonía con el artículo 119 de la Constitución, donde se establece que con el fin de proveer propuestas que no impliquen la ejecución normal de las funciones de las autoridades territoriales, el Estado puede situar recursos adicionales y tomar medidas especiales para al gunas de ellas. El sistema financiero de la capital debería incluir:

a) La aportación de una cantidad por las cargas de gestión y organizativa, cuyo importe está fijado en el uno por mil de los ingresos impositivos del Estado; la obligación para toda la comunidad nacional de soportar las funciones de «SU» capital está claramente resal tada.

b) La financiación del plan de obras públicas de interés nacional en la capital; entre otras cosas, el plan contemplará que otras Administraciones Públicas o el sector privado contribuyan a financiar actuaciones concretas, particularmente a través de la financiación de proyectos.

c) Algunos principios a través de los cuales se desarrollará la autonomía financiera de la ciudad, resolviendo de este modo las dificultades de interpretación derivadas de los artículos 23 y 119.2 de la Constitución. La actuación autónoma se centrará en la ejecución de obras públicas de interés para la ciudad. El coste de las obras públicas de interés nacional habrá de ser sufragado por el Estado.

d) La transferencia de la propiedad del Estado y la riqueza nacional a la ciudad.

La Administración territorial de la capital posee una complejidad inevitable, en la medida en que se han de tener en cuenta las necesidades funcionales del Estado, de la Santa Sede y de las instituciones internacionales con sede en Roma. Por este motivo, es esencial racionalizar los procedimientos estableciendo que los instrumentos para la planificación general y las modificaciones parciales se adoptan por la Asamblea de la capital, de conformidad con los principios generales de la planificación territorial y regional, una vez que se reciba el visto bueno del Comité para Roma, Capital de Italia. El Gobierno y la Re gión pedirán una revisión de las leyes de planificación y las modificaciones, a través de un procedimiento motivado, sólo conforme a los principios generales de la planificación territorial y regional y las necesidades del Estado y de la Región. Las leyes de planificación y las modificaciones habrán de ser aplicadas, en cualquier caso, cuando sean aprobadas nuevamente por la Asamblea de la capital.

Por el tamaño de su territorio y de la población administrada, la capital sólo tendrá dos niveles de gobierno. De acuerdo con las indicaciones constitucionales, la denominación del nivel de la zona amplia debería ser «Ciudad de Roma, Capital de Italia»; para el nivel de proximidad, se usarán las expresiones «M unicipalidad U rbana» (para los municipios que se integren en la ciudad) y «M unicipio» (como el presente estatuto del municipio de Roma, para los distritos inframunicipales descentralizados). D e todos modos, el sistema de la capital será principalmente «un sistema cooperativo» por definición, donde los niveles institucionales colaborarán y se armonizarán. Por lo tanto, una vez que la experiencia iniciada por la Ley 369/1990 de 15 de diciembre, sea desarrollada y corregida, será necesario:

a) Preparar un «Comité para Roma, Capital de Italia» formado por el Presidente del Consejo de M inistros, el Pre sidente de la Región del Lazio y por el Alcalde de la ciudad. El Comité decidirá los asuntos por mayoría, pero el Presidente del Consejo de Ministros tendrá que dar siempre su voto positivo.

b) Disponer que el Alcalde de la capital debería convertirse en un miembro de derecho de la Conferencia Estado/ Ciudades y autonomías locales y debería ser invitado a las sesiones de la C onferencia Estado/Regiones Autónomas y Provincias.

c) Disponer que, junto con los presidentes de las regiones con un estatuto autónomo especial, el Alcalde de la capi- 
tal debería ser invitado a las sesiones del Consejo de $\mathrm{M} \mathrm{i-}$ nistros cuando se estén tratando asuntos que afecten a la capital.

El Comité para Roma, Capital de Italia, será el lugar para armonizar las funciones de la responsabilidad de la ciudad, del Estado y de la Región, especialmente a través de:

a) El informe sobre las reglas de la capital que derogan regulaciones legislativas del Estado y de la Región.

b) El informe sobre la planificación urbanística de la capital.

c) El informe y propuestas sobre el plan de obras públicas de interés nacional dentro del territorio de la capital.

d) La aprobación del plan para localizar las sedes de las Administraciones Públicas nacional y regional y sus órganos dentro del territorio de la capital.

e) La decisión final en los foros para la provisión de servicios, cuando la objeción de una Administración responsable de la protección del medio ambiente, el paisaje y el territorio, y la conservación del patrimonio histórico y artístico o la protección de la salud no se supere.

Con el fin de asegurar la máxima eficiencia de las funciones de la competencia local conectadas al peculiar estatuto internacional de Roma, sería aconsejable la presencia del Alcalde de la capital en los órganos bilaterales que el Estado italiano establezca con la Ciudad del Vaticano, así como la creación de delegaciones permanentes de la ciudad de las organizaciones internacionales con sede en Roma. Al mismo tiempo, con el fin de crear una red efectiva entre la ciudad y las instituciones de la Unión Europea, es necesario prever:

a) La presencia por derecho del Alcalde de la ciudad entre los miembros ordinarios del «Comité de las Regiones» que son designados en representación de Italia.

b) La creación de una oficina de representación ante las instituciones de la Unión Europea. c) La introducción de las propuestas de la ciudad en los programas nacionales y en las correspondientes iniciativas de la Unión Europea en relación con los fondos estructurales.

d) La participación de la ciudad en las fases «ascendente» y «descendente» de las políticas de la Unión Europea, de acuerdo con las especificaciones del «Libro Blanco de la Gobernanza Europea» de 25 de julio de 2001.

¿Será posible al canzar estos objetivos en los próximos meses? Resulta difícil afirmarlo. La iniciativa del Presidente de la Región del Lazio, Francesco Storace, se ha sumado a la tradicional oposición de la Lega N ord (Liga N orte). El señor Storace ha movilizado a todo su partido político - Alleanza N azionale (Alianza Nacional) - para apoyar una línea diferente a la que contempla la inmediata implementación de la nueva regulación constitucional, y se ha centrado en una nueva reforma que otorga a la capital un sistema jurídico de tipo regional. Esta propuesta ha sido proclamada en actos políticamente comprometedores, pero no ha sido nunca transformada en un hipótesis estructurada, por lo que es difícil entender sus propósitos, ¿llegará Roma a convertirse en un «distrito federal» totalmente sustraído de la Región del Lazio? ¿Permanecerá, por el contrario, dentro de la Región, pero con una potestad autónoma de elaboración de leyes para al gunas materias? El último supuesto no parece ser diferente (sólo en importantes aspectos de técnicas reguladoras) de la propuesta del Alcalde. La primera y más radical hipótesis colisionaría con la situación de la Región del Lazio, que tiene más del 70 por ciento de su «peso» en Roma (la población residente y trabajadora, el producto interior bruto doméstico, los servicios a los ciudadanos y las empresas). La Región del Lazio, al igual que otras regiones italianas, está elaborando un nuevo estatuto: es improbable que la «N ueva Constitución del Lazio» sea aprobada sin resolver un asunto tan importante para su futuro. La mayoría de las fuerzas políticas y económicas de la ciudad y los empleados públicos parecen apostar por la futura ley para el sistema de la capital. ¿Será ésta la enésima promesa vacía?
* Director G eneral de la Provincia de Roma.

La traducción al español de la versión original en inglés ha sido realizada por Pedro
Luis Pérez Guerrero, Profesor del Área de Ciencia Política y de la Administración de la Universidad de Alicante. 